\title{
Leaders
}

\section{Heat stress, thermoregulation, and fluid balance in women}

As with so many aspects of exercise related research, most investigations have focused on the male of the species. In 1940, however, Hardy and Du Bois ${ }^{1}$ undertook an investigation into gender related differences in thermoregulation, and indeed the focus on comparing the responses of men and women has dominated this area of research since. Menstrual cycle effects on thermoregulation have probably formed the next major component of work involving women and thermoregulation.

Women generally have a large surface area to mass ratio, relatively great adiposity, and a menstrual cycle. All these properties may influence thermoregulation with regard to its effectiveness and therefore may affect heat tolerance. Core temperature in the luteal phase of the menstrual cycle is higher than in the follicular phase, at least in non-heat acclimated women. ${ }^{2}$ With this, the threshold for sweating onset is elevated and there is a greater skin blood flow. Despite this knowledge, the mechanism for these changes has not been elucidated with any certainty, but it seems that the practical exercise thermoregulatory effects due to the menstrual cycle are minimal in heat stress. ${ }^{3}$

The large surface area to mass ratio of women gives faster heat exchange with the environment while the adiposity alters thermoregulatory effectiveness. The ability to prevent major shifts in core temperature is reduced with heat stress but thermoregulatory effectiveness is increased in the cold. The core temperature of a woman increases more on average than a man's when exposed to equal climatic heat conditions at rest ${ }^{4}$ and during exercise. ${ }^{5}$ The major reasons for this reduced thermoregulatory effectiveness are that in women the onset of sweating occurs at a higher core temperature ${ }^{6}$ and the rate of sweat production is lower than in men, ${ }^{5}$ despite having a greater number of heat activated sweat glands per unit surface area as well as an absolute greater number than men. ${ }^{7}$ It is unclear, however, whether this effect is caused by sex hormones, differences in metabolism of sweat glands, differences in the anatomy of sweat glands, or some other factor(s). The limited information on sweat composition suggests that there seems to be no major gender differences in this area. An elevated iron concentration in female sweat has been reported, ${ }^{8}$ but absolute losses of iron are not different between the genders because of the smaller volume losses in women.

Exercise tolerance time in the heat is less in women than men, ${ }^{9}$ certainly for unacclimatised individuals, possibly because of either excessive heat storage or the relatively low cardiac output. With acclimatisation, however, women's exercise heat tolerance time increases to a greater extent than men's with heat acclimation, ${ }^{10}$ removing a significant portion of the gender difference. This probably occurs because of greater circulatory changes rather than greater evaporative heat loss.

The inter-relation between thermoregulation and body fluid balance stems from the use of evaporative cooling, particularly in response to environmental or exercise induced heat stress. Because of a woman's lower body water content relative to her body mass, any given body water loss expressed as a percentage of body mass is greater than that of a man of the same mass with a lower body fat content. Little work on the fluid balance implications of women exercising in the heat has been undertaken. However, in terms of recovery of whole fluid balance status after a bout of exercise in the heat, the available evidence suggests that, for women who do not retain fluid over the course of their menstrual cycle, there is no difference in the recovery of fluid balance status across the cycle. ${ }^{11}$ There is also evidence to indicate that the rate and extent of dehydration is unaffected by the stage of the menstrual cycle.

In conclusion therefore, much of the literature on female thermoregulation to date is confounded by variables such as body mass and composition, aerobic power, training, and menstrual status. The effect of gender on temperature regulation during exercise must be evaluated when the metabolic heat stress for the two genders is equated. This, however, brings with it a new set of problems of investigating an unrepresentative female population.

For future research, the influence of an altering hormonal status and greater body fat content should provide sufficient stimulus to study the exercising woman in her own right. Despite this enticement, it is probably inevitable that, for most issues in the field of exercise science, the basic physiological understanding will be sought by investigations on men without their complicating factors, in a physiological sense at least!

Biomedical Sciences, University Medical School,

SUSAN M SHIRREFFS

Foresterhill, Aberdeen AB25 2ZD, Scotland

1 Hardy JD, Du Bois EF. Differences between men and women in their responses to heat and cold. Proc Natl Acad Sci USA 1940;26:389-98.

2 Carpenter AJ, Nunneley SA. Endogenous hormones subtly alter women's response to heat stress. F Appl Physiol 1988;65:2313-17.

3 Stephenson LA, Kolka MA. Effects of gender, circadian period and sleep loss on thermal responses during exercise. In: Pandolf KB, Sawka MN, Gonzalez RR, eds. Human perfomance physiology and environmental medicine at terrestrial extremes. Indianapolis: Benchmark Press, 1988:267-304.

4 Bittel J, Henane R. Comparison of thermal exchanges in men and women under neutral and hot conditions. F Physiol (Lond) 1975;250:475-89.

5 Wyndham $\mathrm{CH}$, Morrison JF, Williams CG. Heat reactions of male and female Caucasians. F Appl Physiol 1965;20:357-64.

6 Cunningham DJ, Stolwijk JAJ, Wenger CB. Comparative thermoregulatory responses of resting men and women. $\mathcal{f}$ Appl Physiol 1978;45:908-25.

7 Bar-Or O, Lundegren M, Magnusson LI, et al. Distribution of heat-activated sweat glands in obese and lean men and women. Hum Biol 1968;40:23548.

8 Lamanca JJ, Haymes EM, Daly JA, et al. Sweat iron loss of male and female runners during exercise. Int f Sports Med 1988;9:52-5.

9 Frye AJ, Kamon E, Webb M. Responses of menstrual women, amenorrheal women, and men to exercise in a hot, dry environment. Eur f Appl Physiol 1982;48:279-88.

10 Frye AJ, Kamon E. Responses to dry heat of men and women with similar aerobic capacities. F Appl Physiol 1981;50:65-70.

11 Maughan RJ, McArthur M, Shirreffs SM. Influence of menstrual status on fluid replacement after exercise-induced dehydration in untrained healthy young women. Br F Sports Med 1996;30:41-7. 


\section{Food for thought}

An anonymous referee once advised me to be less hesitant with my conclusions when, with cautious optimism, I wrote, "It is suggested that there is a possibility that glutamine might have a beneficial effect in certain circumstances."

Many manufacturers of supplements have no such caution. "Constituent $\mathrm{X}$ will restore your vitality after exercise!, will stop your muscles aching!, will avoid that heavy legs syndrome!, will improve your immune system etc." One memorable plug included the statement that "Glutamine will cure glandular fever". A letter inquiring about the scientific basis for this elicited no response. It appears easier, in principle, for a company to make money from unsubstantiated claims such as these than it is to obtain ethical approval for experimental research on athletes.

Athletes are regularly advised about good nutrition and a sensible, well balanced diet. The reality is different. They are more likely to have a high protein and high fat but low carbohydrate diet and to take high supplementary doses of more than one vitamin and/or mineral product.

The problem, however, is that the content of many "different" supplements overlaps, and thus the athlete may overdose on some constituents. Responsible manufacturers advise clearly not to take more than the recommended dose-nevertheless, combinations of supplements can lead to overdosing. Tea and coffee can inhibit absorption of iron. Taking a combination of supplements, one of which is iron, with others containing caffeine, may have the opposite effect to that intended owing to the accumulated caffeine content.

Taking $60 \mathrm{mg}$ vitamin $\mathrm{C}$ a day in adults will maintain a body pool of $1500 \mathrm{mg}$ and any excess is likely to be excreted. The ingestion of much higher amounts, perhaps double the recommended daily allowance (RDA) commonly consumed by athletes, may create problems with iron loading, copper absorption, or kidney oxalate stone formation in susceptible people.

For vitamin A, not only is there no advantage in ingesting more than the RDA but also vitamin $A$ is toxic in very high doses. There are anecdotal reports of supplements that contain a dosage substantially in excess of the RDA of some vitamins. It is easy to say that athletes should not be taken in by "charlatans", but it is difficult for athletes to discern which companies are giving sensible advice in the midst of sophisticated marketing techniques.

A brief survey of scientific publications since 1966 shows that eight supplementation research studies on vitamins and minerals were carried out, including three on iron, and two or three on protein or carbohydrate ingestion between 1966 and 1986. In the succeeding four years interest in vitamins and minerals expanded, as reflected by 16 studies, and reports on carnitine, dihydroxyacetone pyruvate, and inosine also appeared. Between 1991 and 1996 interest in iron increased, and studies on carnitine expanded; vitamins and minerals were still of interest with more than 20 studies reported; carbohydrate was back in fashion (10 studies); and a newcomer, creatine, became very popular, with seven studies carried out. In the past two years, another 11 creatine studies have been published, with carbohydrate (six) and iron still of interest. These figures are approximate, and do not include glutamine and branched chain amino acid supplementation, which will be dealt with separately.

The main aim of creatine supplementation is to increase the levels of phosphocreatine (a major fuel to be used in explosive sports): it is also used to increase muscle mass. Many athletes have reported problems associated with its use, including muscle cramps and gastrointestinal disorders. However, as yet, there is no scientific evidence to substantiate these claims; equally, there is no research into the long term effects of high doses of creatine.

Carbohydrate boosting-"carbo-loading"-is a well known regimen followed by many marathon runners since it was first mooted many years ago, and the major pre-marathon pasta parties attract a huge number of runners. That carbohydrate should provide $60 \%$ of energy is well known, but many athletes still do not take sufficient during either training or competition.

It has been claimed by some research workers that provision of glutamine or a precursor reduces the incidence of infections after prolonged, exhaustive exercise. ${ }^{12}$ However, the precise effect on immunodepression has yet to be established. ${ }^{34}$

Supplementation of the branched chain amino acids, such as leucine, isoleucine, and valine (BCAA), has been widely studied for its effect on central fatigue, which emanates from the brain, rather than peripheral fatigue, which emanates from muscle. The mental exertion necessary to maintain a given power output is extremely important in central fatigue, and BCAA supplementation has been found to improve mental performance after prolonged, exhaustive exercise. ${ }^{5}$

For the most part, there seems to be no substitute for a healthy, balanced diet, and a "quick fix" is seldom appropriate. However, an athlete's interpretation of what constitutes a balanced diet may be uncertain. We must continue to emphasise the importance of a high carbohydrate intake for both training and competition, but it is difficult to wean some athletes away from the notion that supplementation is the answer to all their problems. Nevertheless, supplementation may be advantageous in some situations. Ideally, athletes should have easy access to impartial advice on the value of supplementation in their own individual circumstances.

University Department of Biochemistry,

LINDA M CASTELL

South Parks Road,

Oxford OX1 $3 Q U$

1 Castell LM, Poortmans JR, Newsholme EA. Does glutamine have a role in reducing infections in athletes? Eur F Appl Physiol 1996;73:488-90.

2 Bassit RA, Sawada LA, Bacurau, RFP, et al. The effect of BCAA supplementation upon the immune response of triathletes. Int $\mathcal{F}$ Sports Med (in press)

3 Rohde T, MacLean DA, Pedersen BK. Effect of glutamine supplementation on changes in the immune system induced by repeated exercise. Med Sci Sports Exerc 1998;30:856-62.

4 Rohde T, Asp S, MacLean DA, et al. Competitive sustained exercise in humans, lymphokine activated killer cell activity, and glutamine - an intervention study. Eur f Appl Physiol 1998;78:448-53.

5 Blomstrand E, Hassmen P, Ek S, et al. Influence of ingesting a solution of branched-chain amino acids on perceived exertion during exercise. Acta Physiol Scand 1997;159:41-9. 


\section{Exercise and primary dysmenorrhoea}

In the last 15 to 20 years, research into the link between physical activity and menstrual disorders has increased significantly. Exercise has been found to affect menstruation in a variety of ways, including inducing amenorrhoea in athletes and perhaps alleviating symptoms involved in premenstrual syndrome and dysmenorrhoea. Primary dysmenorrhoea is characterized by a range of symptoms, foremost of which are lower abdominal pain that may radiate to the lower back or legs, headache, nausea, and vomiting. Some $75-85 \%$ of women experiencing dysmenorrhoea report symptoms as mild, although dysmenorrhoea may cause significant absence from school and work. The symptoms are thought to stem from elevated levels of prostaglandins resulting in uterine contractions and ischaemia. The origin of the increase in prostaglandins has not been decisively identified; one likely mechanism involves a decline in progesterone in the premenstrual phase, which results in the synthesis of prostaglandins in endometrial cells by membrane phospholipids. This theory is supported by the success of prostaglandin synthesis inhibitors in pain relief. However, as these inhibitors only provide pain relief in $70-75 \%$ of women, other factors are probably also involved. Possible contributors include deficient degradation of prostaglandins as the result of a defect in prostaglandin dehydrogenase, the vasocontrictive action of antidiuretic hormone, and variations in pelvic blood flow, which may influence synthesis or breakdown of prostaglandins. Primary dysmenorrhoea affects between 47 and $80 \%$ of the general population depending on the age group studied, as it tends to decrease with age and after pregnancy. ${ }^{1}$

Although exercise is generally thought to alleviate the discomfort associated with dysmenorrhoea, the scientific literature on this phenomenon displays mixed evidence. One study shows a decline in the severity of symptoms after a 12 week aerobic training programme, ${ }^{2}$ and another reports diminished dysmenorrhoea in exercising junior high school girls. ${ }^{3}$ Similarly, women who train intensively have been found to report fewer symptoms than women who exercise occasionally. ${ }^{4}$ However, a number of other studies have failed to find any relation between dysmenorrhoea and levels of physical activity. ${ }^{5-7}$ Furthermore, Metheny and $\mathrm{Smith}^{8}$ report that, after controlling for disposition and mood, exercise is actually associated with higher levels of menstrual discomfort.

An interesting element of the relation between exercise and dysmenorrhoea is the involvement of stress. A number of studies have shown a correlation between life stress and gynaecological symptoms. Increased levels of cortisol have been associated with premenstrual syndrome, ${ }^{9}$ and one study showed a correlation between menopausal symptoms and environmental stress. ${ }^{10}$ Furthermore, dysmenorrhoea has been treated successfully with stress reduction techniques. ${ }^{11}{ }^{12}$ Exercise is widely accepted as a means of moderating stress and stress related symptoms, perhaps by reducing the perception of stress and/or biochemical changes in the immune system. ${ }^{13} \mathrm{~A}$ mechanism by which exercise may improve the symptoms of dysmenorrhoea by reducing stress has been articulated by Gannon. ${ }^{13}$ Menstrual pain probably stems from increased contraction of the uterine muscle, which is innervated by the sympathetic nervous system. Stress tends to enhance sympathetic activity, and may therefore increase menstrual pain by exacerbating uterine contraction. By relieving stress, exercise may decrease this sympathetic activity, thereby alleviating symptoms.

However, as exercise has repeatedly been shown to improve mood, reduced dysmenorrhoea may simply result from enhanced mood, as opposed to actual alleviation of physical symptoms. In fact, exercise is known to cause the release of endorphins, substances produced by the brain that raise the pain threshold. Many studies fail to control for disposition and mood; the controlled study of Metheny and $\mathrm{Smith}^{8}$ suggests that, although exercise may alleviate stress induced dysmenorrhoea, it may simultaneously aggravate symptoms. One explanation of this aggravation may be that exercise raises somatic awareness, as regular exercise has been associated with increased sensitivity to bodily states. Alternatively, if exercising women reduce their activity levels in the premenstrual phase, the resulting fall in endorphin levels could intensify dysmenorrhoea. ${ }^{8}$

Overall, research into the relation between dysmenorrhoea and exercise has been hampered by methodological flaws such as varying definitions of dysmenorrhoea and activity, different modes of data collection, disparate study design, failure to perform blind studies, and retrospective reporting of symptoms. Blind studies are particularly important in this area of research, as it has been shown that women may be influenced by previously held beliefs, taboos, and social expectations involving menstruation when answering menstrual distress questionnaires. ${ }^{14}$ This complication, along with the confounding effects of stress, points to the variety of factors influencing dysmenorrhoea and its relation to exercise. More carefully controlled longitudinal studies should help elucidate this complex relationship. Research in this area is important given the high prevalence of dysmenorrhoea and the potential benefits of exercise.

REBECCA J LOCKE MICHELLE P WARREN

Department of Obstetrics and Gynecology, Columbia University, New York, NY, USA

1 Warren MP, Shangold MM. Sports gynecology: problems and care of the athletic female. Cambridge, MA: Blackwell Science, 1997.

2 Israel RG, Sutton M, O'Brien KF. Effects of aerobic training on primary dysmenorrhea symptomatology in college females. $\mathcal{F}$ Am Coll Health 1985; 33:241-4.

3 Golub L, Menduke H, Lang WR. Exercise and dysmenorrhea in young teenagers: a 3-year study. Obstet Gynecol 1968;32:508-11.

4 Izzo A, Labriola D. Dysmenorroea and sports activities in adolescents. Clin Exp Obstet Gynecol 1991;18:109-16.

5 Harlow SD, Park M. Physical activity is not associated with any pain parameter. Br F Obstet Gynaecol 1996;103:1134-42.

6 Jarrett M, Heitkemper MM, Shaver JF. Symptoms and self-care strategies in women with and without dysmenorrhea. Health Care for Women International 1995;16:167-78.

$7 \mathrm{Ng}$ TP, Tan NCK, Wansaicheong GKL. A prevalence study of dysmenorrhoea in female residents aged 15-54 years in Clementi Town, Singapore. Ann Acad Med Singapore 1992;21:323-7.

8 Metheny WP, Smith RP. The relationship among exercise, stress, and primary dysmenorrhea. $\mathcal{F}$ Behav Med 1989;12:569-86.

9 Watts JFF, Butt WR, Edwards RL, et al. Hormonal studies in women with premenstrual tension. Br f Obstet Gynaecol 1985;92:247-55.

10 Gannon LR. Menstrual disorders and menopause: biological, psychological, and cultural research. New York: Praeger, 1985.

11 Cox DJ, Meyer RG. Behavioral treatment parameters with primary dysmenorrhea. Ұ Behav Med 1978;1:297-310.

12 Quillen MA, Denney DR. Self-control of dysmenorrheic symptoms through pain management training. $\mathcal{F}$ Behav Ther Exp Psychiatry 1982;13:85-104.

13 Gannon $\mathrm{L}$. The potential role of exercise in the alleviation of menstrual disorders and menopausal symptoms: a theoretical synthesis of recent research. Women Health 1988;14:105-27.

14 Golomb LM, Solidum AA, Warren MP. Primary dysmenorrhea and physical activity. Med Sci Sports Exerc 1998;30:906-9. 


\section{Bone metabolism in exercise associated amenorrhoea: the importance of nutrition}

Osteoporosis was shown to be a health risk for young women with exercise associated amenorrhoea in the early 1980s when a series of studies showed that such women had a lower bone mineral density (BMD) than age matched, active, or sedentary eumenorrhoeic women. ${ }^{1-3}$ Subsequent research showed that active amenorrhoeic women continued to lose bone while their menstrual disturbance persisted, but that their BMD stabilised or increased if their menses resumed. ${ }^{4}$ Furthermore, retrospective analyses of the menstrual histories of these women showed that their BMD correlated positively with the duration for which they had experienced regular menses. ${ }^{5}$

The "traditional" explanation for premature bone loss in young women with exercise associated amenorrhoea has been an oestrogen deficiency. ${ }^{1-5}$ Indeed, the rationale for conducting the aforementioned studies was that it was known that the amenorrhoea of these women coincided with an oestrogen deficiency and that an oestrogen deficiency is the principal cause of bone loss in women with ovarian failure. ${ }^{67}$ Thus it was hypothesised that active amenorrhoeic women may also be at risk of osteoporosis. ${ }^{1-3}$ The results of these early studies not only supported a link between exercise associated amenorrhoea, oestrogen deficiency, and bone loss but, perhaps more importantly, they showed that the exercise undertaken by these active amenorrhoeic women was unable to protect their bone mass.

However, more recent research has challenged the notion that an oestrogen deficiency is the primary cause of bone loss in active amenorrhoeic women. Firstly, these women appear to be less responsive to exogenous oestrogen treatment than women with ovarian failure, for whom oestrogen replacement can offer complete protection against bone loss. ${ }^{6}$ Secondly, studies of bone turnover in women with exercise associated amenorrhoea using biochemical markers of bone cell activities and collagen turnover have suggested that their pattern of bone remodelling is atypical of an oestrogen deficient state. ${ }^{89}$ This state is usually characterised by increased bone turnover with "excessive" bone resorption, which is normalised by oestrogen replacement. ${ }^{67}$ However, studies in our laboratories have shown an apparent reduction in bone turnover and, more especially, reduced bone formation in women distance runners with chronic amenorrhoea (longer than four years) when compared with eumenorrhoeic runners or age matched sedentary eumenorrhoeic women. ${ }^{8}$ It was particularly noteworthy that none of these amenorrhoeic runners exhibited signs of "excessive" bone resorption.

As these amenorrhoeic runners were significantly lighter and leaner than the eumenorrhoeic runners or sedentary women, we explored relations between serum or urine levels of bone turnover markers and indices of nutritional status in these women. We found that serum levels of bone formation markers correlated positively with estimated energy balance, body mass index (BMI), and serum levels of the nutritional markers 3,5,3'-tri-iodothyronine $\left(\mathrm{T}_{3}\right)$ and insulin-like growth factor I (IGF-I) in the amenorrhoeic group of runners, but not in the eumenorrhoeic groups. ${ }^{9}$ The lowest levels of bone formation markers were measured in those amenorrhoeic runners who exhibited characteristic signs of undernutrition, such as a BMI < $17.5 \mathrm{~kg} / \mathrm{m}^{2}$ and a subnormal serum level of $\mathrm{T}_{3}$ and IGF-I. There was no correlation between urine levels of bone resorption markers and any of these nutritional indices.
These findings suggest that undernutrition may underly the bone remodelling imbalance and bone loss in active amenorrhoeic women, and, furthermore, that nutritional factors may counteract or override the stimulatory effects of an oestrogen deficiency on bone turnover.

Previous research has also supported a link between undernutrition, reduced bone formation, and bone loss in young women. For example, women with anorexia nervosa, who are typically undernourished and amenorrhoeic, have reduced bone formation and experience rapid bone loss. ${ }^{10}{ }^{11}$ However, with refeeding, they exhibit an increase in the serum level of bone formation markers ${ }^{12}$ and often a gradual increase in BMD, even without a resumption of menses. ${ }^{10}$ Also, protein-energy malnutrition appears to play a pertinent role in the development of osteoporosis in the elderly and in individuals with malabsorption disorders. ${ }^{13}$ Furthermore, it has been hypothesised that in active women amenorrhoea itself is induced by a chronic negative energy balance, or its effects on body mass, body composition, and metabolism. ${ }^{14}$

Although the metabolic interaction between undernutrition and imbalanced bone remodelling requires further exploration, it is nevertheless known that an acute or chronic energy deficit elicits metabolic aberrations which can lead to inadequate bone formation or excessive bone resorption. ${ }^{15}{ }^{16}$ These metabolic aberrations include hypercortisolaemia, low $\mathrm{T}_{3}$ syndrome, and IGF-I deficiency, ${ }^{15}$ all of which have been documented in active amenorrhoeic women. ${ }^{8915}$ Of interest, in a recent study, short term fasting (four days) in healthy eumenorrhoeic women was shown to elicit a reduction in the serum level of bone formation markers (as well as IGF-I), which was rectified by raising the serum level of IGF-I in spite of continued fasting. ${ }^{16}$ Also, administration of recombinant human IGF-I to anorexic women has been shown to effect a dose related increase in the serum level of bone formation markers. ${ }^{11}$ Thus the correction of metabolic abnormalities that impact on bone formation, such as IGF-I deficiency, is a promising therapy for a nutritionally linked bone remodelling imbalance.

In summary, there is growing evidence to suggest that undernutrition and its metabolic consequences are responsible for precipitating a bone remodelling imbalance which may lead to bone loss in young women with exercise associated amenorrhoea. This evidence does not preclude a role for oestrogen deficiency in the aetiology of this bone remodelling imbalance, as undernutrition is also a suggested cause of ovarian suppression in these women. ${ }^{14}$ The potential to reverse osteopenia in women with exercise associated amenorrhoea is currently debatable, as a normalisation of $\mathrm{BMD}$ has yet to be demonstrated in prospective studies. As reduced bone formation (rather than increased resorption) has been shown in these women, ${ }^{89}$ therapeutic strategies aimed at increasing osteoblast activity and collagen formation are likely to be of greater benefit than agents that retard osteoclast activity. However, as with other types of osteoporosis, prophylaxis is likely to outweigh the benefits of treatment for these women. ${ }^{6713}$ This in turn depends on the treatment of exercise or dietary behaviour which may precipitate a bone remodelling balance.

C L ZANKER

De Montford University, Bedford MK40 2BZ, United Kingdom 
1 Cann CE, Martin MC, Genant HK, et al. Decreased spinal mineral content of amenorrheic women. $7 A M A$ 1984;251:626-9.

2 Drinkwater $\mathrm{BL}$, Nilson $\mathrm{K}$, Chestnut III CH, et al. Bone mineral content of amenorrheic and eumenorrheic athletes. N Engl f Med 1984:311:277-81. 3 Marcus R, Cann C, Madvig P, et al. Menstrual function and bone mass in elite distance runners. Ann Intern Med 1985;102:158-63.

4 Drinkwater BL, Nilson K, Ott S, et al. Bone mineral density after resumption of menses in amenorrhoeic athletes. FAMA 1986;256:380-2.

5 Micklesfield LK, Lambert EV, Fataar AB, et al. Bone mineral density in mature, pre-menopausal ultramarathon runners. Med Sci Sports Exerc 1995;27:688-96.

6 Hergenroeder AC. Bone mineralisation, hypothalamic amenorrhoea and sex steroid therapy in female adolescents and young adults. F Pediatr 1995;126: 683-9.

7 Manolagos SC, Jilka RL. Bone marrow, cytokines and bone remodelling: emerging insights into the pathophysiology of osteoporosis. $N$ Engl f Med 1995;332:305-11.

8 Zanker CL, Swaine IL. Bone turnover in amenorrhoeic and eumenorrhoeic distance runners. Scand 7 Med Sci Sports 1998;8:20-6.

9 Zanker CL, Swaine IL. The relationship between bone turnover, oestradiol and energy balance in women distance runners. Br f Sports Med 1998;32: $167-71$

10 Kreipe RE, Hicks DG, Rosier RN, et al. Preliminary findings on the effects of sex hormones on bone metabolism in anorexia nervosa. 7 Adolesc Health 1993;14:319-24.

11 Grinspoon SK, Baum HB, Lee K, et al. Effects of short-term recombinant human insulin-like growth factor 1 administration on bone turnover in osteopenic women with anorexia nervosa. F Clin Endocrinol Metab 1996;81: 3864-70.

12 Mackintosh C, Abraha H, Stefanis $\mathrm{N}$, et al. Biochemical bone markers in anorexia nervosa [abstract]. Bone 1997;20;34S

13 Heaney RP. Nutritional factors in osteoporosis. Anпи Rev Nutr 1993;13: 287-316.

14 Bonen A. Exercise-induced menstrual cycle changes: a functional, temporary adaptation to metabolic stress. Sports Med 1994;17:373-92.

15 Laughlin GA, Yen SSC. Nutritional and endocrine-metabolic aberrations in amenorrheic athletes. F Clin Endocrinol Metab 1996;81;4301-9.

16 Grinspoon SK, Baum HB, Peterson A, et al. Effects of short-term recombinant human insulin-like growth factor 1 administration on bone recombinant human insulin-like growth factor 1 administration on bone

\section{Child abuse and the sports medicine consultation}

Are doctors who are involved in sports medicine really aware that child abuse in sport may present to them indirectly in the sports injury clinic? How many of the "injuries" or "fatigue producing poor performances" in children are cries for help that are missed in the consultation room? When should we think harder about possible child abuse? Might signs of physical, emotional, or sexual abuse be the effects of abusive relations at home or in sport rather than just the causes of disappointing sports performance?

Sport at the highest level is demanding more time from its participants, many of whom have been involved in training and competition from a very young age. ${ }^{1}$ The closeness of the abuser/athlete relationship could lead to opportunities for either the paedophile or the sexual predator $^{2}$ to indulge in unacceptable physical contact or emotional abuse of the athlete. Although all gender permutations have been reported in accounts of sexual abuse in sport, most known cases have occurred between an older male authority figure and a younger female athlete (hence the terminology adopted in this article). It is also important to stress that the term "abuse" refers not only to sexual exploitation but may also apply to physical or emotional exploitation or to neglect. ${ }^{3}$

Abusers are diverse in terms of their sociodemographic characteristics and motives. ${ }^{45}$ Most reported abusers in sport have been coaches, but research is still at an early stage and administrators, parents, senior athletes, and medical and paramedical staff, who also have personal contact with athletes, present possible additional risks. Those sports for which cases of abuse are already in the public domain are characterised by a high level of social and personal contact between the coach and athlete.$^{6}$ This, often intense, relationship requires hours of training time together, travel to national and international competitions, close proximity at meals, visits to the coach's home, and often, importantly, shared accommodation. The athlete, over months or years, learns to respect and obey this mentor. She sees him in loco parentis, a confidante, and a shoulder to cry on. The abusing coach uses this dependence, creating a climate of favouritism, secrecy, and closeness with malice aforethought. ${ }^{2}$ This process is called "grooming" and is planned to secure complete secrecy, co-operation, and compliance from the targeted athlete.

Although folklore suggests that certain sports are more prone to problems of sexual exploitation than others, it is highly dangerous to focus on just a few. All sports are risk settings; certainly the degree of risk varies from one setting to another but many risks are also shared across sports. On the basis of current research data, it is not possible to say with certainty exactly which sports pose the greatest risks of sexual exploitation to the athlete. However, preliminary analysis suggests that the stage just before peak performance, what Brackenridge and Kirby ${ }^{1}$ call the "stage of imminent achievement", is the time when the athlete has both the most to lose by leaving the sport and everything to gain by complying with the demands of authority figures around her. If the stage of imminent achievement coincides with puberty, then the risks are thought to be greater: in other words, early peaking sports are thought to present the greatest risks of exploitation by sexual predators in authority positions.

It is not uncommon for the athlete to wait several years before disclosing experiences of abuse. The reasons behind this are complex. The athlete usually fears that the coach may victimise or harass her if she withdraws her co-operation or, indeed, that her own athlete peers may resent her, either for receiving favouritism during the abuse or for upsetting the dynamics in the club, team, or squad. It is not unknown for a coach to sexually abuse several athletes at the same time. In one case, a paedophile coach regularly abused more than 30 boys in a community club, and in another an elite coach pursued predatory sex with two separate athletes in his squad of only four young girls. These two athletes did not discover this until several years afterwards. Anyone disclosing abuse risks personal stigma and other severe consequences by putting this knowledge into the public domain. Most frightening of all to the young aspiring medal winner is the prospect of losing favour from the coach and being dropped by him from the squad.

Although many sports have introduced vetting and monitoring procedures for their coaches, at all levels, by no means all have done so. The sports medicine specialist, among others, has the opportunity to identify early signs of inappropriate distress in athletes that may indicate abuse related problems. ${ }^{7}$ Doctors and physiotherapists should check the child protection and reporting procedures for their own sports teams or governing bodies to ensure that the athlete and themselves are protected. Unfortunately police data on potential child abusers in sport are flawed. Official registers record only those people with convictions and some for whom there is significant evidence but no conviction. However, until the proposed new Criminal Records Bureau becomes operational, access to these data continues to be strictly controlled and to exclude most voluntary sector groups. 
The vast majority of abusers in sport evade detection or continue to operate behind a wall of silence and approval because they are good coaches and/or appear to be "nice men". Sexual abuse is characteristically covert and secretive. Perpetrators prey on the vulnerable in their care, usually targeting athletes who are vulnerable and suffering low self esteem. If suspicion is aroused, the abuser may move on to another sport or to another part of the country. The best treatment of child abuse is, of course, prevention. For the abuser to feel threatened he must know there is an environment within his sport where child abuse is talked about openly. There should be meetings with athletes, officials, and medical staff to establish good practice, and both athletes and their parents must know that there are channels within the sport for reporting harassment.

The next time the symptoms and signs don't really add up in the consultation, or the young athlete's demeanor is subdued and inhibited, or she shows signs of disordered eating, or the adult in charge is a little too domineeringthink twice.

Practical guidance on child protection and antiharassment procedures for sport organisations is available from Professor Celia Brackenridge. Email: brackenridge@chelt.ac.uk.

ROD JAQUES

Winfield Hospital, Gloucester

CELIA BRACKENRIDGE

Cheltenham and Gloucester College of $\mathrm{HE}$

1 Brackenridge $\mathrm{CH}$, Kirby S. Playing safe: assessing the risk of sexual abuse to elite child athletes International Review for the Sociology of Sport 1997;32:407-18.

2 Brackenridge $\mathrm{CH}$. Sexual harassment and sexual abuse in sport. In: Clarke G, Humberstone B, eds. Researching women and sport. London: Macmillan, 1997:126-41.

3 Crouch M. Protecting children: a guide to sportspeople. Leeds: National Coaching Foundation/NSPCC, 1995.

4 Finkelhor D, et al, eds. A sourcebook on child sexual abuse. London: Sage, 1986.

5 Morrison T, Erooga M, Beckett RC. Sexual offending against children: assessment and treatment of male abusers. London: Routledge, 1994

6 Brackenridge $\mathrm{CH}$. "He owned me basically...": women's experience of sexual abuse in sport. International Review for the Sociology of Sport 1997;32: $115-30$.

7 Brackenridge $\mathrm{CH}$, Kirby S. Protecting athletes from sexual abuse in sport: how theory can improve practice. In: Lidor R, Bar Eli M, eds. Innovations in sport psychology: linking theory and practice. Morgantown, West Virginia: Fitness Information Technology, 1999. 